\title{
A Realistic Technique to Obtain the Surface Contours of Conventional, Thickened and Paste Tailings Storage Facilities
}

J. Engels, D. Dixon-Hardy, B. Birch Department of Mining, Quarry and Mineral Engineering, University of Leeds, United Kingdon

\section{INTRODUCTION}

Understanding the surface fluctuations of a dry stack, paste, thickened and conventional tailings storage facility can help manage the day to day tailings disposal operations. This in turn helps to increase the safety of the facility and reduce the hazards associated with a failure. The majority of conventional tailings failures arise from poor water management, most notably loss of freeboard. Controlling the geometry of the supernatant pond and the freeboard of a conventional tailings facility are perhaps the fundamental parameters influencing embankment stability. Although dry stack, paste and thickened tailings facilities don't have to control supernatant water as stringently, there is a need to control the geometrical properties of the facility.

Calculating the contours of a tailings facility accurately can help give a better insight into how the facility is performing and how it will perform in the future. This is perhaps better presented by highlighting the advantages to attaining elevation data:

Conventional surface impoundments:

- Areas that are susceptible to loss of freeboard can be determined.

- The area and volume of the supernatant pond can be calculated accurately.

- The available volume of tailings that can be stored at current embankment elevations can be determined.

- Depth of water around decant towers and barges can be determined.

- The expected geometry of the supernatant pond if there is an elevation change (e.g. storm, decant system failure) can be calculated.

- The flow paths from individual spigots can be determined.

- Beach widths and angles can be measured.

- Variable consolidation rates can be assessed.

- Areas of internal erosion or foundation settlement (e.g. piping, sink hole development) can be identified. 
Dry stack, paste, thickened impoundments:

- The area and volume of the stacked material can be calculated.

- The likely flow paths of the tailings discharging from the spigots can be identified.

- Calculation of the likely zoning timescales and covering of desiccated areas is possible.

- The slope angles of the deposit can be measured.

- The areas around the perimeter where the bleed water will collect can be identified.

It is important to have a high accuracy of elevation data as the majority of tailings facilities cover large areas which can cause a significant error to water management calculations. This is particularly true for conventional tailings facilities where the beach angles are shallow and there is a significant distance between the discharge points and the decant facility.

Determining elevation data of a tailings facility can be achieved by a variety of methods. This paper summarises various methods of obtaining elevation data of the surface of a tailings facility quickly, easily, accurately and in a cost effective way. The idea behind this research is to establish highly accurate data to allow 3D models to be generated which can be modelled with GIS software. These models will be discussed and a few examples given to demonstrate how knowing elevation data can help to improve the management of a tailings storage facility. One of the most notable outcomes of these models will be the ability to predict supernatant pond geometry, the flow paths and accumulations from multiple discharge points.

\section{HISTORIC AND CURRENT ATTEMPTS TO ACCESSING LOW BEARING CAPACITY TAILINGS}

Technology is available today to allow access onto tailings in both an easy and safe way. Amphibious or low ground bearing vehicles have been used on tailings to collect samples, make visual inspections, chase away bird life, install piezometers and wicks, compact tailings in hydraulic cells and plant reed beds. The most common vehicle used is the hovercraft due to its low cost and versatility. A small handful of mines either own or have used a hovercraft on their tailings facilities.

The Australian Centre for Geomechanics (ACG) modified a small hovercraft to house a drill rig with the aim of collecting and testing in-situ tailings material. They reported to have had no problems with the craft and could penetrate material to $4 \mathrm{MPa}$ with a Cone Penetration Test (CPT) without problems (Newson, 1997). However, ACG did discover some safety issues that need to be considered when using any vehicle on tailings. Perhaps the most important issue is what happens if the engine fails or the vehicle becomes stuck in soft tailings? A backup vehicle is the obvious solution but if that fails a major problem can unfold. The ACG had a 'harpoon' system in place that fired a rope out to the impoundment perimeter that would allow a larger 
rope to be connected for towing the craft in. ACG's modified craft with and without the drill rig attached is shown in Figure 1 and 2 respectively.
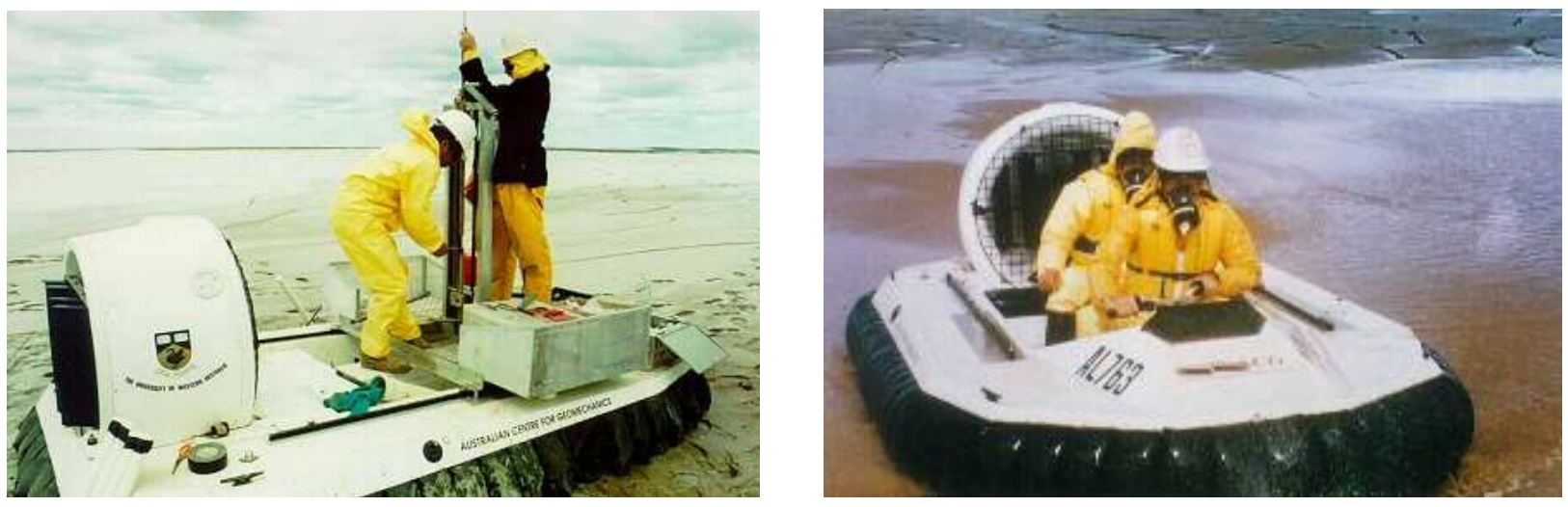

Figure 1 and 2 Hovercraft used to collect samples from soft areas of tailings

Barricks Goldstrike operation in the Nevada desert have used hovercraft and a tracked swamp buggy to access the soft tailings, shown in Figures 3 and 4. Barrick and Vector Engineering installed wick drains into soft tailings with the swamp buggy in an attempt to dissipate pore pressures and increase consolidation rates as well as increase the overall available storage volume of the impoundment.
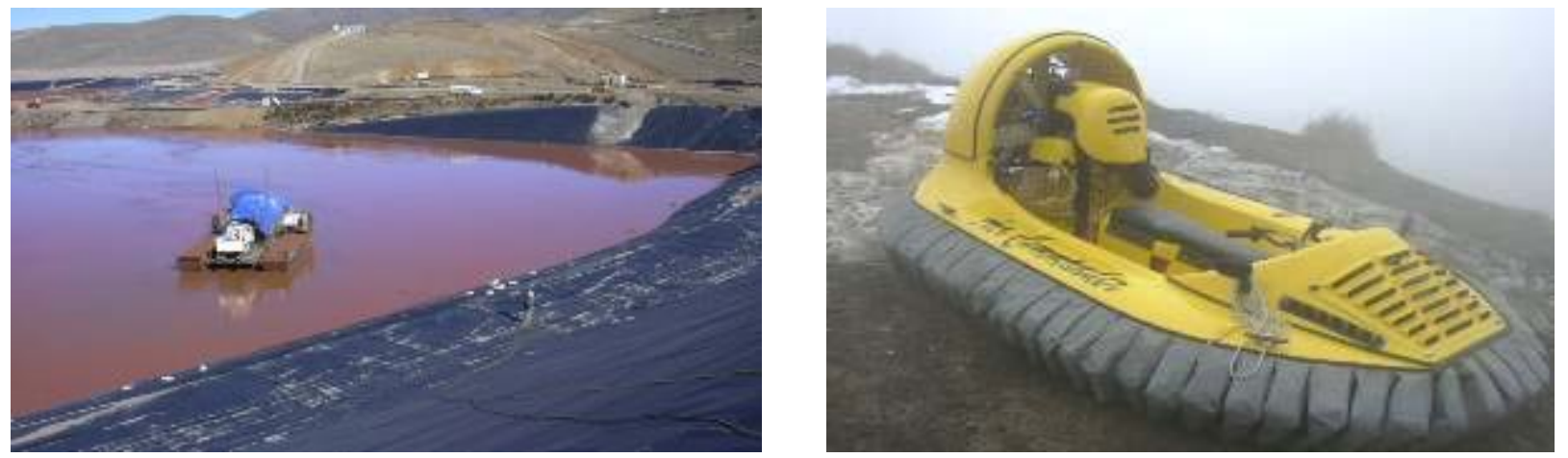

Figure 3 and 4 Swamp buggy and one of the hovercrafts used at Goldstrike

The equipment used on the tailings was very successful and the high weight of the swamp buggy allowed the wicks to be installed to a greater depth than what could be expected when using a hovercraft. Barrick found that the hovercrafts were faster and more versatile than the swamp buggy but generated a considerable amount of dust and spray. This can have health hazards if toxic materials are stored in the impoundment (e.g. raw sewage, cyanide).

Newmont's Carlin operation, also in the Nevada desert, use a fan boat to scare off birdlife and inspect the soft tailings. The fan boat cannot travel through or over dried consolidated tailings and can only be used where standing water is present. On one occasion the engine failed and the operators had no choice but to swim to the edge of the supernatant pond. This strengthens the safety issues that are apparent when accessing tailings. 
Rio Tinto's Northparks mine in Australia use a hovercraft to prevent birds from stopping off at their tailings pond as they migrate. Northparks uses the hovercraft to chase away the birds that have landed on the supernatant pond. A few mines in Canada have also tried this approach to bird control.

\section{METHODS AND TECHNOLOGY AVAILABLE}

This research has looked into a range of different cost effective techniques to obtain accurate elevation data of tailings facilities. Once the data is collected, computer software is used to generate a 3D model of the tailings facility that displays the elevation data as a series of contours. The equipment used to collect the elevation data will $\log$ hundreds of coordinate points that the computer software interpolates as a workable platform. There are various powerful mapping software packages available to interact with the collected data most of which are common on any mine site (e.g. Vulcan, Surpac, ArcGis). The software used for this paper is ESRI's ArcGis 9 which is perhaps a too powerful package for obtaining the information we require but is a familiar program to use for the main author.

This section highlights the various remote sensing and on tailings techniques to collect elevation data.

\subsection{Collecting the elevation data - remote sensing}

Remote sensing is the preferable method of collecting the elevation data of a tailings facility as the risks associated with accessing the tailings are eliminated. However, the major disadvantages of remote sensing techniques are the accuracy and cost, making them a less viable option. The following sections highlight the various remote sensing techniques available.

\subsubsection{Photogrammetry}

Photogrammetry is the technique of measuring 2D and 3D objects from photogrammes (photographs, images) to obtain coordinates of the required object points, topographical and thematical maps and rectified photographs (orthophoto). Topographical data and Digital Terrain Models (DTM) are generally obtained from aerial or satellite images that have point references on the ground. This data can be collected from either single or multiple images and either manually interpreted by optical rectification or by using analytical techniques.

Photogrammetry for tailings storage deposits is feasible but to gain the accuracy required the images have to be taken from above ground. As tailings deposits generally cover a large area of land and with conventional storage facilities having relatively flat topography (excluding the embankments) then it is very difficult to attain elevation data from ground level. This means regular flyovers at high elevations would be required (dependent on the size of the tailings facility) to capture the entire area. Segments can be taken and pieced together but this increases the error of the results, particularly for conventional storage facilities that have subtle elevation changes compared to thickened and paste storage. 


\subsubsection{LIDAR}

LIght Detection And Ranging (LIDAR) equipment uses lasers to measure distances to reflective surfaces. Infrared beams are fired at a rate of over 1000/second from the equipment that is typically mounted on the underside of an aircraft. The beams reflect off the target (ground) back to the detector on the equipment. The travel time of the beams is calculated and converted to distance (based on speed of light). The accuracy of the beams depends on the height of the aircraft which also influences the area of coverage that the laser can scan (Harding, 2000).

LIDAR produces high density and high resolution data ( $<1$ metre) with vertical errors ranging from $15 \mathrm{~cm}$ or less (Zhang, Jian, 2002). One consideration to the vertical accuracy is that over the entire area of a tailings facility the LIDAR equipment would collect all the data in a matter of seconds. The actual error of all the readings maybe $15 \mathrm{~cm}$ or less but the actual error between the readings taken within the tailings area should not be as great. This is due to the LIDAR equipment scanning the tailings facility in such a short time frame referencing data to adjacent points. The true elevation of the tailings in relation to actual ground benchmarks isn't required, only the elevation differences of the tailings within the facility is necessary.

For tailings facilities LIDAR would be an excellent method of collecting elevation data accurately and quickly without having to access the tailings. LIDAR would be able to collect far more data points than any manual survey which would lead to more defined contour data. However, cost is the obvious disadvantage, as well as getting the equipment to mine sites which are often in low populated or remote areas.

The Kidd Creek Metallurgical site in Timmins, Ontario, Canada is the location of the worlds first thickened mine tailings facility (Robinsky cone). LIDAR equipment was hired and sent up in a helicopter to collect the surface elevation data of the tailings facility. Unfortunately, the equipment was damaged and no data could be collected. Ten months later Kidd Creek and Golder Associates successfully collected LIDAR data and are currently processing the results. In addition to LIDAR the tailings facility is manually surveyed across accessible areas in an attempt to calculate surface contours.

\subsubsection{Dirigible}

The research carried out for this paper looked into using a dirigible (blimp) as a method of remote sensing a tailings facility. The initial idea was to use an auto-scanning reflectorless profiling laser hanging beneath a blimp that could be towed by a vehicle as it drives around the perimeter of a conventional tailings facility. The anticipated data would give an accurate 3D profile of the embankment, its crest and part of the tailings beach. This would then help a designer to understand crest elevation differences as well as establish if the embankments had moved compared to a previous survey.

Tests were carried out using a tethered blimp and it was quickly realised that a steady path would be difficult to hold even in minor wind conditions. Another problem would be trying to synchronise the profiling laser with a GPS system to obtain accurate ground coordinates. The end result would be a cheaper and less 
accurate version of LIDAR that would be hard to control. The weight of the equipment and its power source also posed a problem as a larger blimp would be required which would increase stability but increase the cost of filling the blimp with helium. Figures 5 and 6 shows the blimp in action with the ground based unit manually tracking its movements.
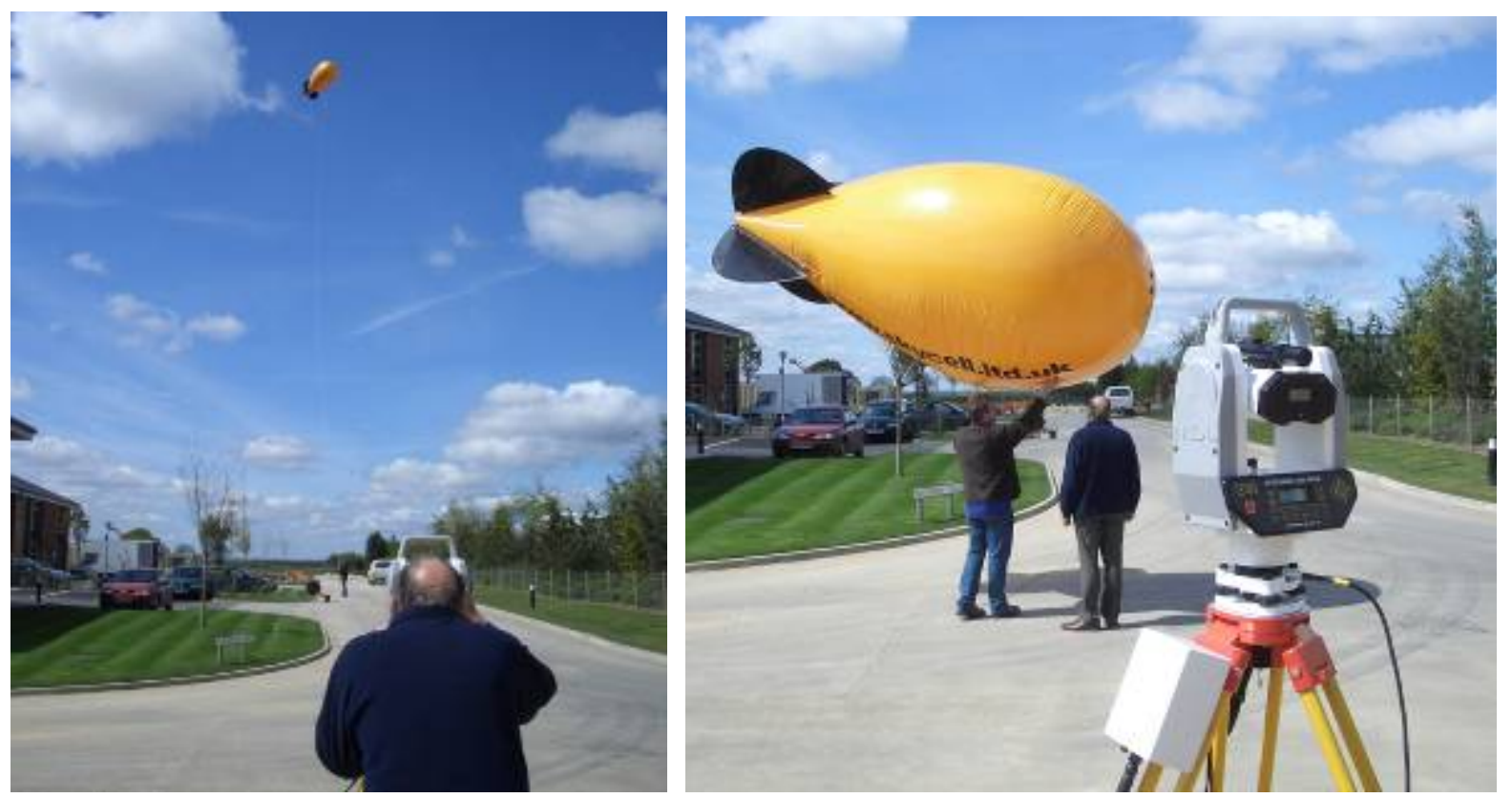

\section{Figure 5 and 6 Blimp testing with the MDL ground based unit}

\subsection{Collecting the elevation data - on tailings techniques}

Accessing tailings to obtain elevation data is far more hazardous than remote sensing techniques but if done properly risks can be reduced dramatically. Chapter 2 already highlighted the various attempts and methods used today to access tailings at a range of different mine sites. At this stage it is clear that the most effective method of accessing tailings is by using a hovercraft which is also the least expensive method.

As mentioned in Section 2 there are other advantages to accessing tailings which make the "on tailings" techniques more preferable than remote sensing.

\subsubsection{Tracking the movement of a vehicle on tailings}

The first step is to establish the equipment that will be used to collect the elevation data of the tailings facility by the vehicle on the tailings. GPS is an obvious choice as it can operate independently to base stations and log thousands of data entries as the vehicle travels over the surface of the tailings facility. GPS is also relatively inexpensive and is common to any mine site with a tailings facility anywhere in the world. The only drawback with GPS is the vertical accuracy which on the majority of GPS equipment is never stated whereas horizontal accuracy is. The simple reason for this is mainly down to the position of the satellites that the GPS receiver obtains its coordinates from. Even when four or more satellites are used to compute a position, the vertical position component of a GPS receiver is usually less accurate than the 
horizontal components. When vertical accuracy is not explicitly stated on GPS equipment, a good general guide is to assume that the vertical accuracy is always two to three times worse than the horizontal (Gilbert, 1997). This vertical accuracy can be reduced by using a base station placed on a known location that sends real time error corrections to GPS receivers. If a connection is lost with the base station the GPS receiver switches back to the satellites and the vertical (and horizontal) errors increase. Although GPS is easy to use, accuracy is somewhat unpredictable particularly in the vertical axis (' $z$ ' component).

A better alternative to GPS with the aim of improving the vertical accuracy is to use a conventional theodolite to survey a point on the vehicle. Surveying by conventional methods can be precise if the equipment is setup correctly on a known point and has reference points. Infrared total stations can be used to calculate the coordinates of a point from a known base station point and can be just as accurate as a conventional theodolite. The total station can also be robotic and automatically track the movement of a reflector. These types of total stations are again common place on any mine site and are relatively inexpensive to buy. It is also possible to upgrade a modern total station to be fully motorised and robotic at a very reasonable cost.

It is therefore proposed that a vehicle's movement be automatically tracked by a robotic total station that is using a reflective $360^{\circ}$ prism mounted on the vehicle. This prism will have to be placed on the tallest point of the vehicle to allow it to manoeuvre without restricting the line of sight between the total station located at the edge of the tailings facility and the prism on the vehicle. It is envisaged that the vehicle will have a lightweight pole affixed to the front of the unit that will be raised off the surface of the tailings. The pole will have a float at its base with an in built sonar system to measure the distance between the float and the tailings. The pole and float will be on a gimbal system to take into account gradient changes which will ensure the pole maintains its vertical axis thus reducing the error of the results.

The sonar system will have to be synchronised with the total station prior to data collection to ensure continuity between the two automated systems. The reason for the sonar is to prevent any contact with the tailings which may adversely affect the vehicle performance whilst negotiating soft areas. The sonar, float and pole configuration also allows data collection of the depth to tailings when on the supernatant pond of a conventional facility. The pole can simply be lowered to allow the float with the built in sonar sensor to rest on the pond allowing depth data to be collected. Establishing these depths will help to build up a more accurate picture of volumes of tailings and supernatant water stored in a conventional impoundment. It also allows the depth to tailings around decant facilities to be established thus optimising the positioning of barges to increase the clarity of decant water.

\subsubsection{Hovercraft}

This paper proposes research to adapt a small hovercraft to be able to survey a tailings facility both quickly and accurately and in a safe and acceptable manner. Modifications to the actual workings of the hovercraft are not required as any 'off the shelf' hovercraft is capable of travelling over tailings without any problems. 
In fact the only modifications to the hovercraft that are required to allow it to survey a tailings facility are very simple. The hovercraft can be mounted with the lightweight pole in front of the craft to prevent any data interference as a result of the downward force of the fan displacing any material as the craft passes over the tailings. The hovercraft then simply travels towards and away from the robotic total station logging the data.

\subsubsection{Swamp machine}

Newmont's Carlin Mine and Barrick's Goldstrike mine have also tried using a tracked swamp machine to access their tailings. They used the machine to install piezometers into areas of soft tailings and found that the machine had no problem accessing any part of their tailings facility. The only drawback is that the machine moves very slowly over the tailings and is more difficult to control than a hovercraft.

A swamp machine is feasible for surveying any type of tailings facility but the low travelling speed of the machine makes it unfavourable for collecting vast quantities of data.

\section{MODELLING AND INTERACTING WITH ELEVATION DATA}

Once the elevation data has been collected, powerful software packages can be used to model and interpret the results. Contours can also be easily derived from spot data $(x, y, z)$ which alone can help to identify the expected surface flow paths within a tailings facility. This is particularly useful for thickened and paste sites where these flow paths would determine how a previous zone can be covered quicker by discharging from certain spigots. This helps to cover areas of sulphide tailings before acid generation can occur. For a conventional facility these flow paths can be a good indication of the expected flow paths from spigots and their impact on controlling the geometry of the supernatant pond. The high resolution contour data for the south impoundment of a fly ash disposal site in the UK is represented in Figure 7.

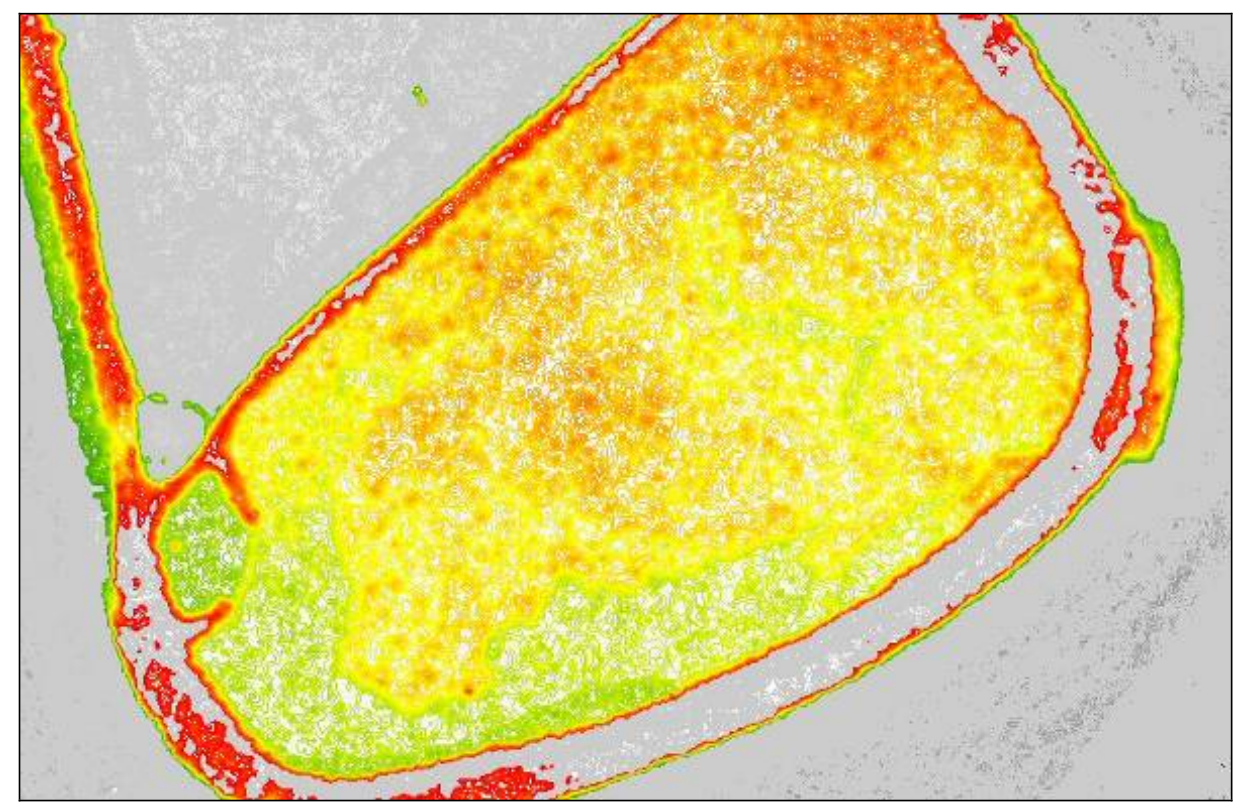

Figure 7 Contours derived from LIDAR elevation data of a fly ash impoundment 
In Figure 7 the contour limits have been set over a $2 \mathrm{~m}$ vertical range with the green areas (around the south and west inner perimeter wall) being the lowest elevation. The LIDAR data that was collected corresponds to the deposition techniques of this particular impoundment at the time.

Using the hydrographical modelling tools of ArcGIS the stream accumulations and flow paths can be determined. These overlays represent the path a drop of water is likely to take over the surface of the impoundment as determined by the elevation data of the model. These flow paths are useful when determining the flow direction and accumulation of tailings that discharge from a particular spigot. These overlays are also useful for determining final capping runoff zones and areas of likely erosion with a view to implementing mitigation measures. Figure 8 shows the flow accumulations and paths for the same impoundment as Figure 7. The thick lines represent the flow directions and accumulations of surface runoff, the dots indicate drainage points (where streams meet) and the dark shaded area is an example of one of the drainage basins within the impoundment.

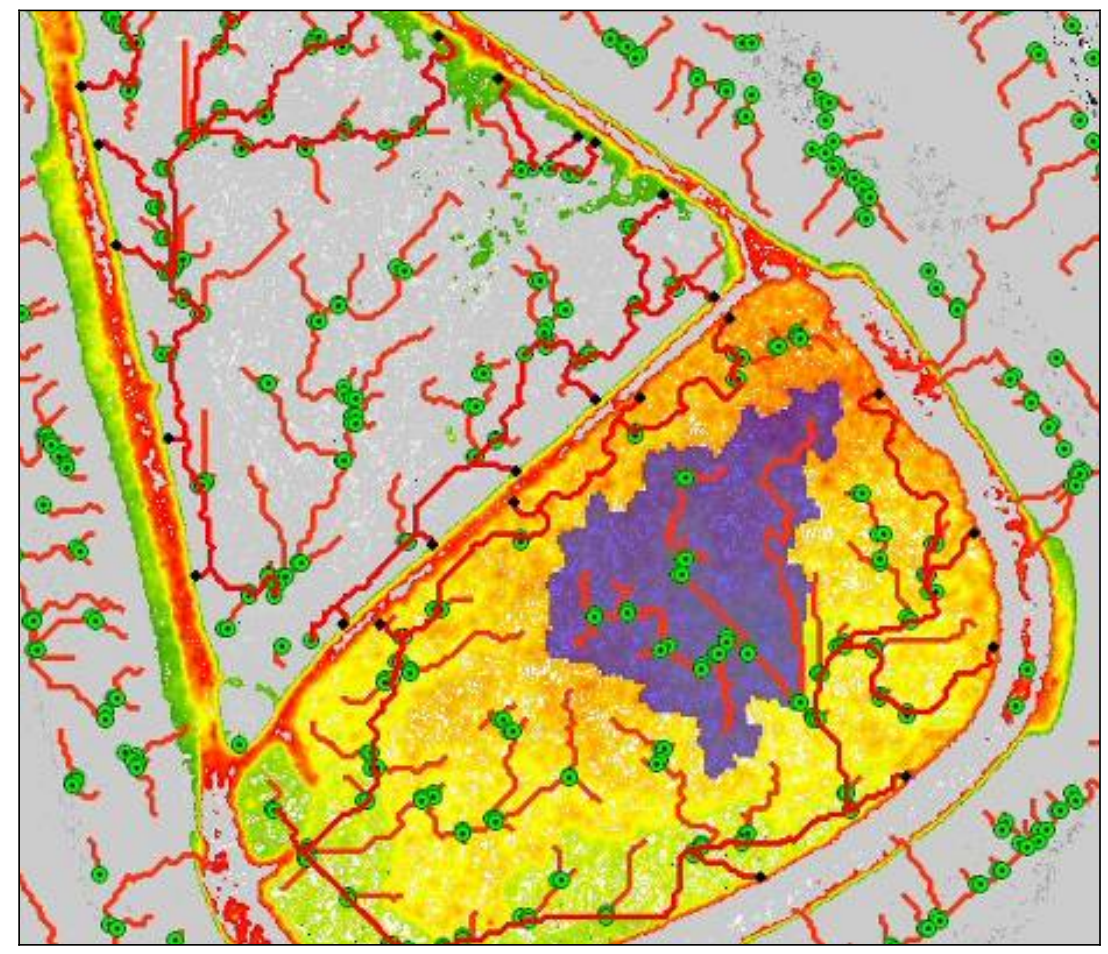

\section{Figure 8 Surface runoff accumulations and flow paths within the waste area}

Figure 9 shows the flow paths from simulated spigots around the perimeter of the north and south impoundments. The flow from any location can be quickly determined by simply clicking on a specific point anywhere within the dataset.

These particular impoundments occasionally store the flushing water from the power stations but on the day the LIDAR data was taken the ponds were dry, otherwise there would have been no reflection from the waters surface (i.e. no data). These discharges generally take the longest routes within the impoundment to increase pond capacity, thus creating the channels around the inner perimeter of both impoundments. A 
picture of the south impoundment holding flushing water is shown in Figure 10. The picture was taken looking across to the decant tower on the opposite side of the embankment.

This explains why the flow paths in Figure 9 meander around the inner walls and flow towards the decant towers in the bottom left of the picture.

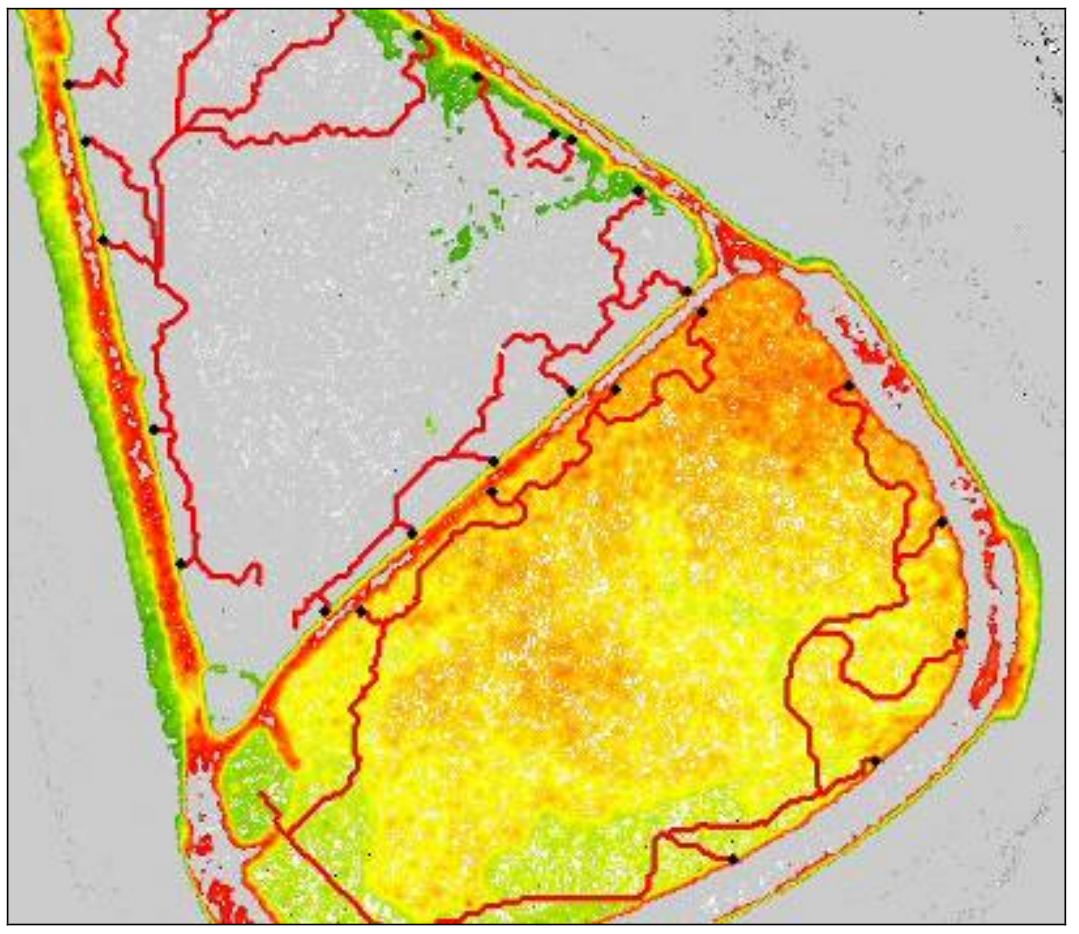

Figure 9 Simulated perimeter spigot flow paths within the fly ash impoundment

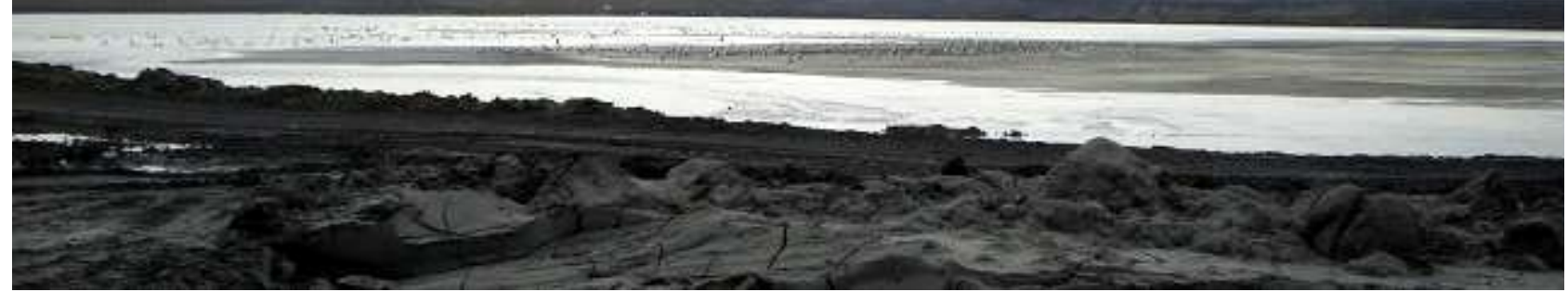

\section{Figure 10 Picture of the south impoundment showing flush water storage zoning}

Cover systems for tailings facilities (and waste rock zones) can be modelled in much the same way. The flow paths and accumulations can be determined to establish the areas most vulnerable to high relief and erosion. The flow paths can also identify where standing water is likely to accumulate during high precipitation and runoff events. Figure 11 shows the runoff flow paths for a fines storage dump after restoration was complete at a coal preparation plant in West Yorkshire. The thick lines indicate point flow paths, the large dots show where standing water is likely to occur and the darker shaded zone highlights a drainage basin. 


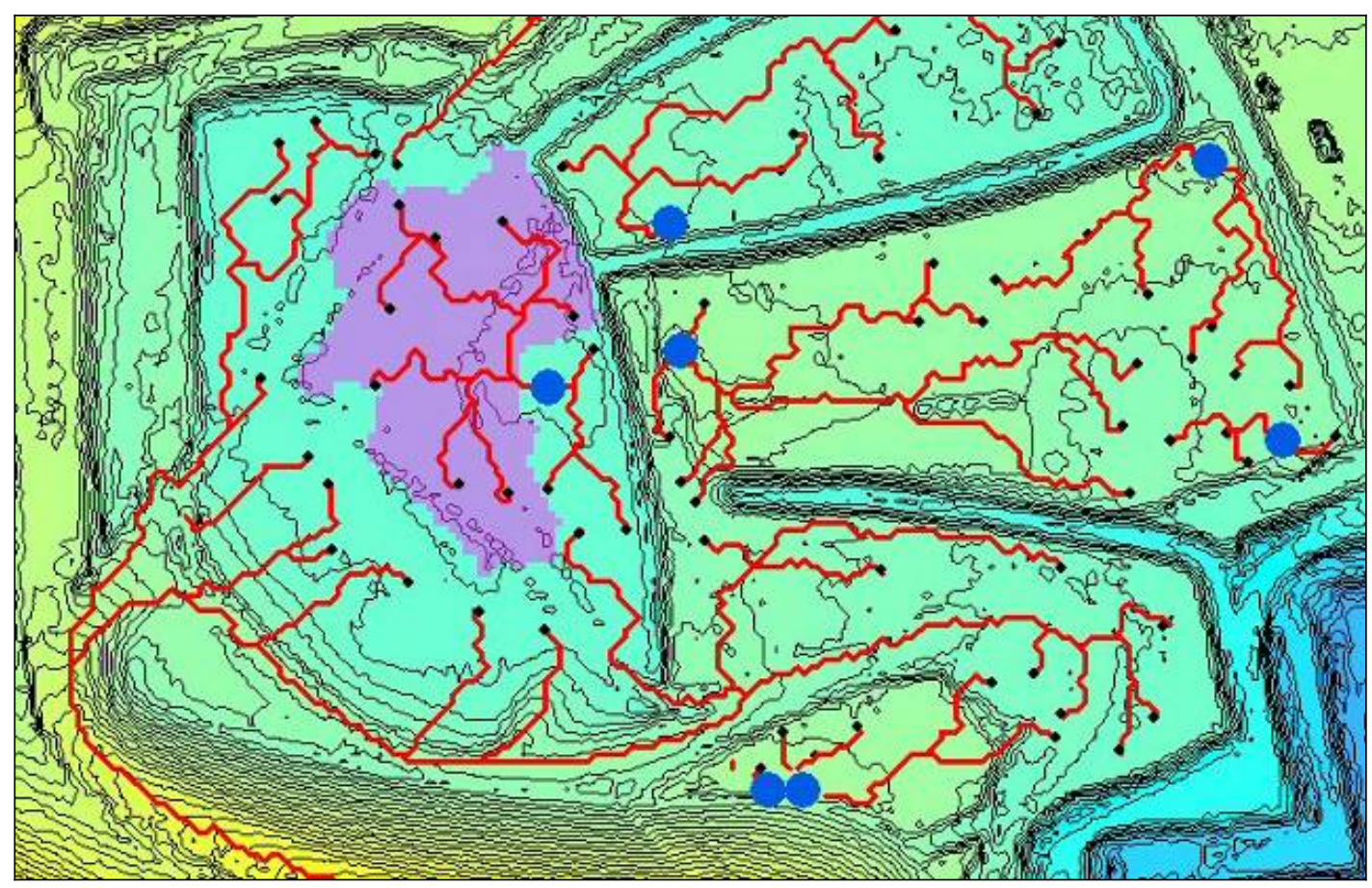

Figure 11 Surface runoff flows, potential water collection zones and watershed analysis

Another advantage to elevation data is that areas and volumes can be calculated easily and accurately. Most importantly the area of the supernatant pond can be determined and the expected geometrical shift if a rise in the pond level occurs (e.g. after a storm). Using the sonar data the volume of the pond can be established as well as the contours underwater. This is more useful to establish the available water capacity of a tailings storage facility at a set beach width or freeboard limit. Water harvesting calculations can easily determine the annual water that is likely to fall within the tailings storage area. Breaking this down to known high rainfall months can help to determine storage capacity and pond geometry. Using the elevation data and the modelling software can help to identify the discharge zoning requirements to prevent loss of freeboard and increase the water storage volume of the facility without jeopardising stability.

Old elevation data can be overlayed with new elevation data to establish the cut/fill areas of the tailings deposit. This is useful for establishing the volumetric changes and where material has deposited within the impoundment (fill). Areas of erosion/channelling can also be identified and their volumes determined (cut).

Figure 12 shows how the elevation data can help to determine the geometry and area of the pond if the water level risers successively in $6 \mathrm{~cm}$ intervals. It can be seen that the geometry of the pond has changed and the locations of short beach widths and low freeboard can be determined. Further analysis of data can calculate the volumes that discharge from specific spigots that would fill particular drainage basins. Hence the time will be able to be determined when the tailings flow will change its meandering path within the facility. Force fill zones can be established by identifying actions that can be taken to dramatically change the current flow paths within the facility. This is particularly useful for thickened and paste storage facilities where temporary riser roads can be created at suitable locations to force fill a particular zone. This might be to 
cover areas of acid generating tailings, push ponded water away from sensitive areas or increase the storage capacity of the facility.

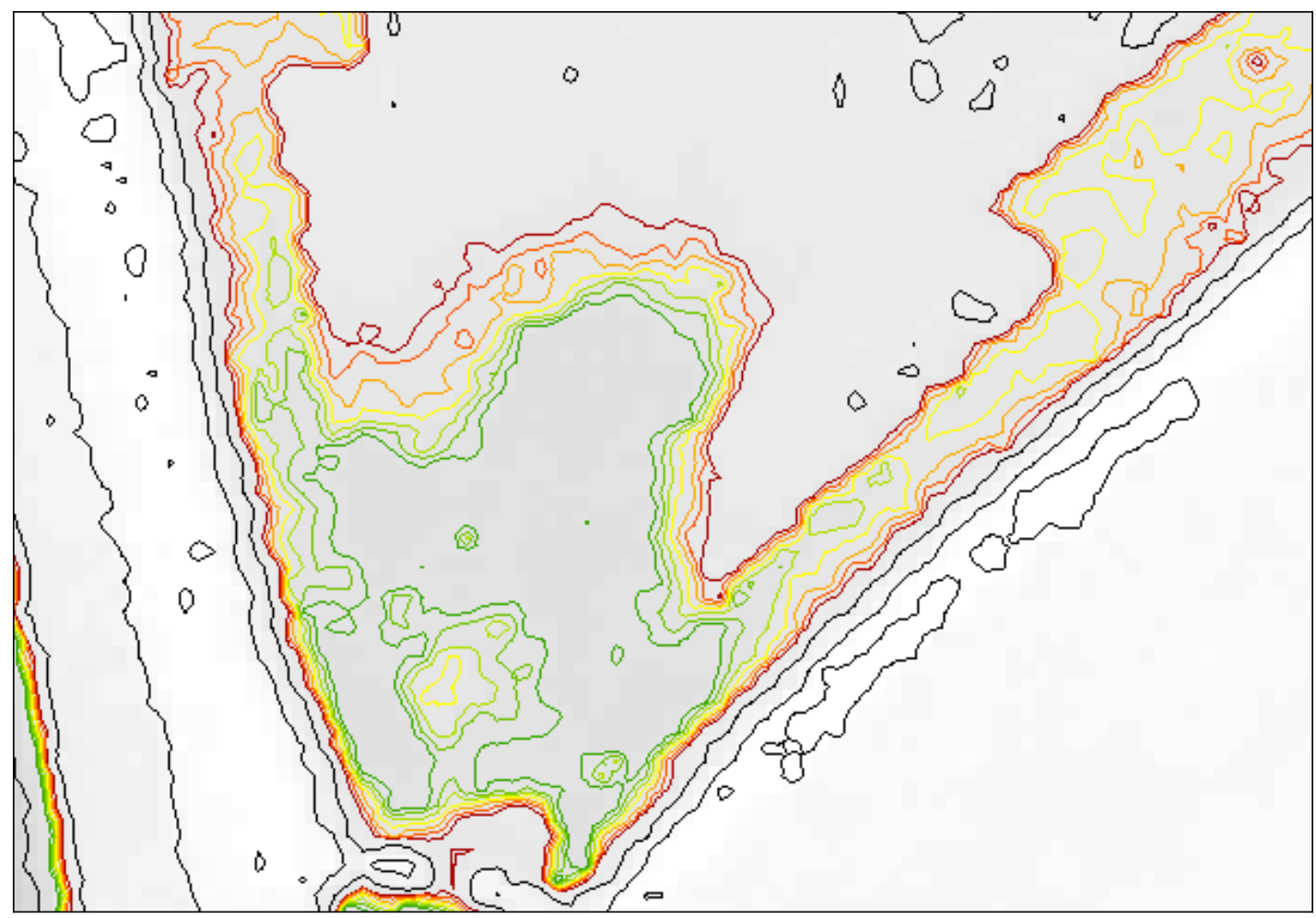

\section{Figure 12 Modelling of the supernatant pond geometry at variable water levels}

\section{CONCLUSION}

This paper has identified and determined realistic techniques for obtaining elevation data of a surface tailings storage facility. The reasoning behind obtaining the data has been discussed as well as the potential outcomes of interacting with the data.

Photogrammetry is appropriate for thickened and paste tailings facilities but the images will have to be taken above the facility to prevent accessing the tailings and to get the full conical shape of the facility into one image. This can be achieved cost effectively by positioning a crane or man lift at points around the tailings facility and using a GPS to determine the exposure locations. Photogrammetry isn't suitable for conventional tailings storage facilities due to the small differences in elevation between the embankment beach and the decant facility. This is particularly true for shallow beach angles where photogrammetry won't provide accurate areas and volumes of the supernatant pond if the levels and geometry were to be predicted.

LIDAR data collected at a low level (preferably by a helicopter to allow slow fly overs) can produce highly accurate and concentrated data that can be directly inputted to just about any GIS or 3D software package. The only drawback with LIDAR is the cost associated with hiring the equipment to carry out the survey. For sites that require irregular surveys LIDAR becomes feasible, particularly considering the accuracy and 
quantity of data that can be attained. However, for the intended outcome of this paper, regular surveys are required to map the ever changing contours of a tailings facility to improve day to day management.

Using a dirigible is feasible but other methods of elevation data collection are far more practical and accurate. The biggest drawback is controlling the blimp to allow a ground unit to track it successfully. This becomes increasingly difficult the further the blimp is away from the ground unit. This leads to another problem in that a radio controlled blimp would be required to access the areas of the tailings facility where no roadways are present.

The use of a hovercraft is far more appealing considering the practicality of being able to use the equipment for other research and work within soft areas of tailings (e.g. wick installation, sample collection). The only drawback with the hovercraft is that the data collection density won't be as high as LIDAR and so the true contour values won't be as precise. Regardless of this the expected data collected will be adequate to successfully model a conventional, thickened, paste and dry stack tailings facility. For conventional storage the hovercraft will be able to collect depth to tailings reading which all remote sensing methods are unable to determine.

Of all the various methods of data collection assessed for this paper LIDAR is the best option for obtaining accurate elevation data of a tailings facility. The high concentration of data and the speed of collection are very appealing. The vertical accuracy is a minor drawback but not that crucial for thickened, paste and dry stack sites as standing water volumes don't influence the day to day management strategies used at the facility. However, LIDAR data is expensive to obtain and for a tailings facility it can be impractical considering the area requiring mapping and the short duration the data will be valid for. The ever changing geometry and conditions of a tailings facility require surveys to be carried out regularly to be able to understand how the facility is performing.

The cost of LIDAR is continually reducing and soon there will be cheaper more robust units for a fraction of the current cost. This would make LIDAR a more attractive method of data collection compared to the hovercraft. It is also inevitable that the vertical accuracy will increase making LIDAR a perfect solution for mapping the earths surface in a quick and easy way.

The power of software is improving and like LIDAR the cost is ever reducing. Various models can be created with the click of a mouse to help better manage a tailings facility. One of the biggest problems with conventional tailings storage is not knowing the inner workings of the facility accurately. Being able to determine future spigot flow paths, available water storage volumes, current tailings and water storage volumes as well as freeboard can help to dramatically reduce the risk of embankment instability. Determining elevation data and modelling it with software generates a better picture of how the facility is performing and how it will perform in the future. This would not only help to prevent failures but also prevents vast environmental damage and even loss of human life which have become associated with tailings impoundment failures. 


\section{REFERENCES}

Air Navigation Order (2000) Statutory Instrument No 2000/1562.

Doneus, M. (1996) Introduction to photogrammetry, University of Vienna, Viewed $20^{\text {th }}$ September 2005 $<$ http://www.univie.ac.at/Luftbildarchiv/wgv/intro.htm>

Gilbert, C. (1997) GPS consumer series: the vertical component of GPS, Earth Observation Magazine May 1997. Viewed 25 September $2005<\mathrm{http}: / /$ www.eomonline.com/Common/Archives/1997may/97may_gilbert.html $>$

Harding, J. (2000) Airborne laser altimeter terrain mapping, NASA's Goddard Space Flight Center, Viewed $5^{\text {th }}$ September 2005 $<$ http://rocky2.ess.washington.edu/data/raster/LIDAR/laser_altimetry_in_brief.pdf $>$

Molander, C., Merritt, S. and Corrubia, A. (2002) Marrying photogrammetry and LIDAR, Earth Observation Magazine June 2002. Viewed $25^{\text {th }}$ September $2005<\mathrm{http} / /$ www.eomonline.com/Common/currentissues/June02/molander.html $>$

Newson. T. and Fahey, M. (1997) Site investigation on active tailings deposits using a hovercraft, Australian Geotechnical Journal, 31, June, pp. 45-55.

Rules of the Air Regulations (1996) Statutory Instrument No 1996/1393.

Surpac $\left(\right.$ Sirovision $\left.{ }^{\mathrm{TM}}\right)$, Digital photogrammetry digital photo imaging, Viewed $25^{\text {th }}$ September 2005 $<$ http://www.surpac.com/products/sirovision/photogrammetry.shtml $>$ 